\title{
Double-armed TMZ-CD40L/4-1BBL Oncolytic Ad5/35 Adenovirus LOAd703
}

National Cancer Institute

\section{Source}

National Cancer Institute. Double-armed TMZ-CD40L/4-1BBL Oncolytic Ad5/35

Adenovirus LOAd703. NCI Thesaurus. Code C148462.

A double-armed oncolytic adenovirus composed of a recombinant genetically modified E1/E3-deleted, adenoviral serotype 5 (Ad5) vector, with the L5 segment of the Ad5 fiber replaced by the shaft and knob from the Ad35 serotype (Ad5/35), which expresses a trimerized (T MZ) form of the membrane-bound immunostimulator CD40 ligand (CD40L; TNFSF5) and the ligand for the signaling domain 4-1BB (4-1BBL; CD137L; TNFSF9), under the control of a CMV promoter, with potential immunostimulating and antineoplastic activities. Upon intratumoral administration of double-armed TMZCD40L/4-1BBL oncolytic Ad5/35 adenovirus LOAd703, the virus infects and selectively replicates in tumor cells. This causes direct oncolysis and the release of a plethora of tumor-associated antigens (TAAs) from the tumor cells. The released TAAs stimulate the immune system and activate anti-tumor cytotoxic T-lymphocytes (CT Ls), thereby further killing tumor cells. In addition, infected tumor cells and tumor stroma cells, including stellate cells and infiltrating immune cells, express the immunostimulants CD40L and 4-1BBL. The expressed CD40L and 4-1BBL proteins activate the CD40L- and 41BBL-mediated signaling pathways, respectively. This activates antigen-presenting cells (APCs), such as dendritic cells (DCs) and M1 macrophages. The DCs produce various cytokines, including interleukin-12 (IL12), IL21, tumor necrosis factor-alpha (TNFa), and interferon-gamma (IFNg), which leads to the activation and expansion of both T-cells and natural killer (NK) cells. Altogether, this modulates immune suppression in the tumor microenvironment (TME), disrupts tumor stroma, and causes additional immune stimulation against tumor cells, thereby inducing further tumor cell lysis. In addition, CD40L reduces myeloid suppressor cells in the TME. Inclusion of the chimeric Ad5/35 fiber targets CD46 and increases viral uptake in cells. Transgene expression is driven by a separate promoter to allow for efficient expression in both tumor cells and tumor stroma. Replication is restricted to tumor cells by the presence of the delta24 mutation in 
the E1A gene, which deletes the retinoplastoma protein (pRb)-binding domain and forces viral replication to be conditional on hyperphosphorylated $\mathrm{pRb}$ and a dysfunctional $\mathrm{Rb}$ pathway. 